\title{
Cobalt-55 positron emission tomography in traumatic brain injury: a pilot study
}

\author{
Hugo M L Jansen, Joukje van der Naalt, Adriaan $H$ van Zomeren, \\ Anne M J Paans, Lammy Veenma-van der Duin, Joffre M Hew, Jan Pruim, \\ Jan M Minderhoud, Jakob Korf
}

\begin{abstract}
Department of Neurology

H M L Jansen

J M Minderhoud

Department of

Biological Psychiatry

H M L Jansen

L Veenma-van der Duin

J Korf

Co-PET showed focal uptake that extended beyond the morphological abnormalities shown by MRI and CT, in brain regions that were actually diagnosed with EEG. Thus Co-PET is potentially useful for diagnostic localisation of both structural and functional abnormalities in moderate traumatic brain injury.
\end{abstract}

Department of Neuro-

Psychology

A H van Zomeren

National PET

Research Center

A M J Paans

J Pruim

Department of NeuroRadiology, Groningen

University Hospital,

PO Box 30.001, 9700

RB Groningen, The

Netherlands

J M Hew

Correspondence to:

Dr Jakob Korf, Departmen

of Biological Psychiatry,

Groningen University

Hospital, PO Box 30.001,

9700 RB Groningen,

The Netherlands.

Received 22 February 1995

and in final revised form

21 August 1995

Accepted 12 September 1995

(F Neurol Neurosurg Psychiatry 1996;60:221-224)

Keywords: traumatic brain injury; PET; cobalt-55

Traumatic brain injury mostly affects young people and can alter physical functioning, cognitive skills such as memory, and personality. ${ }^{1}$ Severity of traumatic brain injury is assessed with the Glasgow coma scale (GCS), which determines the level of consciousness in terms of eye opening, verbal response, and motor response. ${ }^{12}$ Traumatic brain injury is categorised as mild, moderate, or severe depending on this GCS assessment. Even mild (GCS 13-15) traumatic brain injury with transient symptoms can cause delayed neuronal and cellular deterioration and considerable long term deficits. ${ }^{3}$ Moderate traumatic brain injury

\begin{abstract}
Traumatic brain injury is usually assessed with the Glasgow coma scale (GCS), CT, or MRI. After such injury, the injured brain tissue is characterised by calcium mediated neuronal damage and inflammation. Positron emission tomography with the isotope cobalt-55 (Co-PET) as a calcium tracer enables imaging of affected tissue in traumatic brain injury.
\end{abstract}

The aim was to determine whether additional information can be gained by Co-PET in the diagnosis of moderate traumatic brain injury and to assess any prognostic value of Co-PET.

Five patients with recent moderately severe traumatic brain injury were studied. CT was performed on the day of admission, EEG within one week, and MRI and Co-PET within four weeks of injury. Clinical assessment included neurological examination, GCS, neuropsychological testing, and Glasgow outcome scale (GOS) after one year.
(GCS 9-12) often involves lasting, subtle, changes in personality and cognitive performance. ${ }^{3}$ Imaging techniques such as CT, MRI, SPECT, and emission PET have been used to diagnose severity of traumatic brain injury and to predict clinical outcome, showing morphological changes, regional cerebral blood flow ( $\mathrm{rCBF}$ ), and metabolism of the injured brain. ${ }^{4-7}$

Damage by closed traumatic brain injury (without penetration of the skull) can be primary or secondary. Although primary injury (direct mechanical damage to cells and axons at the moment of injury) accounts for the immediate brain damage (acute neuronal death), secondary injury (pathophysiological events as a result of either global or focal ischaemia) arises often from late onset progressive neuronal damage (delayed neuronal death). ${ }^{89}$ Both acute and delayed neuronal death have been attributed to calcium overload and inflammatory processes. ${ }^{910}$

To detect and define traumatic tissue damaged by brain injury we used cobalt -55 with PET (Co-PET) to monitor the calcium mediated damage with PET. We have previously used Co-PET to visualise brain infarcts and multiple sclerosis, independently of integrity of the blood-brain barrier. ${ }^{11} 12$

The present pilot study included five patients with traumatic brain injury of moderate severity who underwent clinical examination, neuropsychological testing, EEG, CT, MRI, and Co-PET.

\section{Patients and methods}

Five patients who sustained closed traumatic brain injury of moderate severity (GCS 9-12 or GCS 13-15 with post-traumatic amnesia for more than three days), subsequently admitted in a six month period to the department of neurology of the Groningen University Hospital were studied. The study was approved by the medical ethics committee of the hospital. Inclusion criteria were age 16-64 years, post-traumatic amnesia of less than 14 days, and written informed consent. Patients with previous traumatic brain injury, other serious disorder, history of neuropsychiatric problems, or chronic substance misuse were excluded. On admission, all patients received an initial neurological examination 
and GCS assessment. Duration of clinical follow up was one year and included neurological examination, neuropsychological testing, and the extended version of the Glasgow outcome scale (GOS). ${ }^{13}$ All five patients (four men, one woman aged 16 to 59 (mean 32) years) had CT on the day of admission, EEG within one week of brain injury, and Co-PET and MRI three to four weeks after traumatic brain injury (depending on the accessibility of the scanner). Another EEG was obtained after one year.

Computed tomography was performed with a Philips Tomoscan 310. Contiguous $6 \mathrm{~mm}$ thick slices were made parallel to the orbitomeatal line. The total CT time did not exceed 20 minutes.

Awake 21 channel EEGs were performed according to the International Ten-Twenty system for 30 minutes. Hyperventilation for two minutes and intermittent photic and auditory stimulation were used as provocative tests. All EEGs were read by an experienced neurophysiologist and special attention was given to focal abnormalities.

Assessment of site and size of the contusional lesion was by MRI by Philips Gyroscan S15 with a $1.5 \mathrm{~T}$ superconducting magnet. Transverse (TR/TE 650/20) $6 \mathrm{~mm}$ thick slices with a $10 \%$ gap were made parallel to the orbitomeatal line. Coronal T2 weighted (TR/TE 2000/40-110) images were made, followed by enhanced $\mathrm{T} 1$ weighted (TR/TE $650 / 20$ ) images after giving $0.1 \mathrm{mmol} / \mathrm{kg}$ gadolinium-DTPA (Magnevist, Schering)

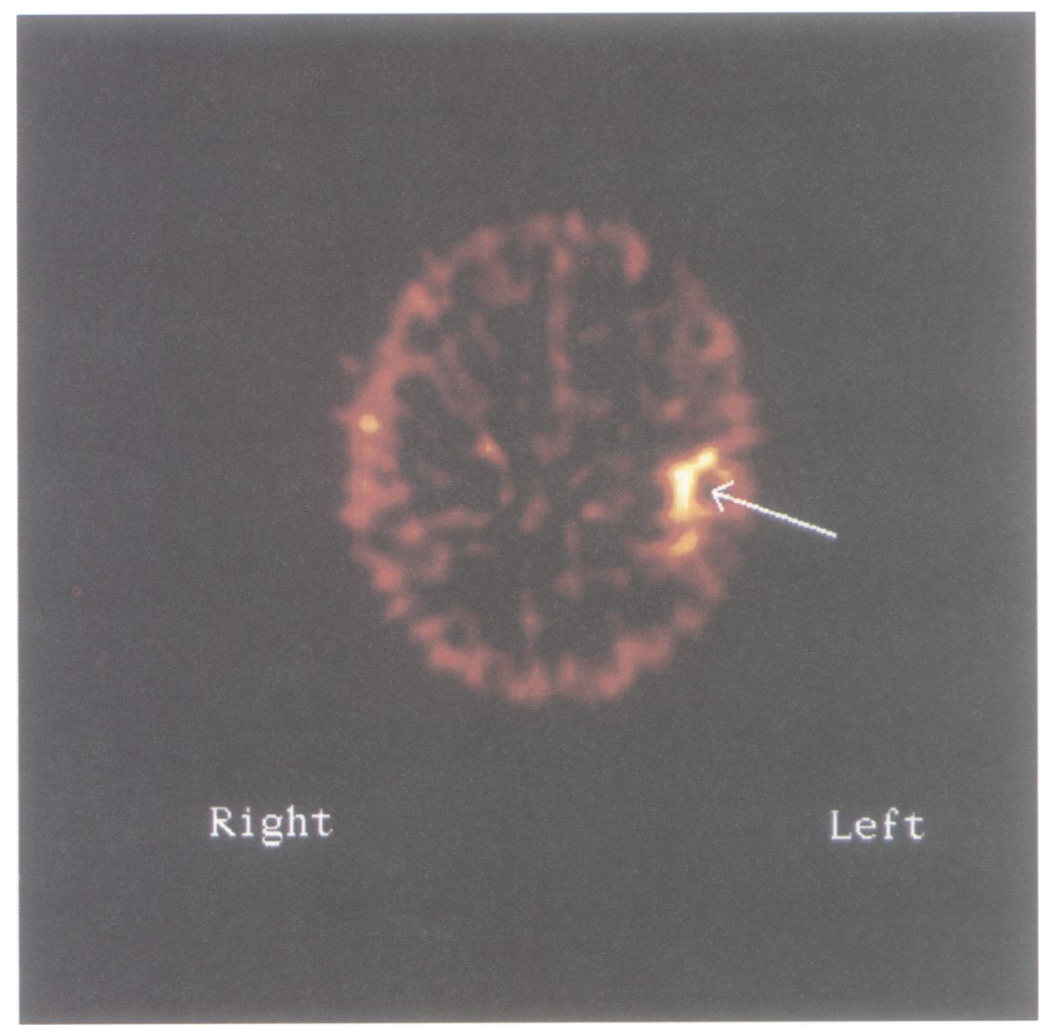

Co-PET of patient $C$ performed 12 days after sustaining a closed traumatic brain injury of moderate severity, disclosing evident Co accumulation in the left temporal region (arrow) which was 3.7 times the activity in the contralateral region. These findings were confirmed with EEG, CT, and MRI, the last showing haemorrhage with slight atrophy. The interval between Co-PET and MRI was 15 days. intravenously. The total MRI time did not exceed 30 minutes. All MR images were assessed by the same neuroradiologist, who had no knowledge of clinical data or Co-PET examination.

The Co-PET was performed in a dynamic mode (six frames of 10 minutes) $20 \pm 4$ hours after intravenous administration of $1 \mathrm{mCi}$ sterile ${ }^{55} \mathrm{CoCl}_{2}$ (Cygne $\mathrm{BV}$, Eindhoven, the Netherlands) using a Siemens ECAT-951/31 positron camera. Contiguous scans were made parallel to the orbitomeatal line and image reconstruction of $3.375 \mathrm{~mm}$ thick planes was done using standard ECAT software (Siemens/CTI, Knoxville, USA). Details of the Co-PET have been described elsewhere. ${ }^{1112}$

To establish a clear delineation of the contusional lesion on Co-PET, the lesion border was outlined as a region of interest by automated contour detection. Subsequently, for each region of interest an identical mirror region of interest was defined in the contralateral hemisphere and the region of interest area and the average cobalt uptake was measured in all (6-10) transaxial planes displaying the traumatic brain injury. Data were combined to obtain the lesion area and the mean average cobalt uptake in the (mirror) lesion. The cobalt enhancement ratio was defined as their quotient. The cobalt contusion load was defined as the product of cobalt enhancement ratio and the lesion area.

Baseline neuropsychological testing was performed between four and six weeks after injury. The test battery applied consisted of the Rey auditory verbal learning test, the Warrington face recognition test, digit span from the WAIS, the Stroop colour word test, trailmaking a and $b$, category fluency (animals and occupations), the paced auditory serial addition task, Wisconsin card sorting test, block designs from the WAIS, and finger tapping. ${ }^{3}$ Assessment was repeated at fixed intervals during follow up-that is, at three, six, and 12 months after injury.

\section{Results}

The table summarises the clinical, CT, EEG, MRI, and Co-PET data. In all patients neuropsychological impairments were found four to six weeks after injury, deficits in verbal and non-verbal memory being the most common.

In patient $\mathrm{A}$, brain abnormalities were evident on Co-PET and EEG. This patient recovered well and had no persistent EEG abnormalities after one year. Patient B had abnormalities on Co-PET, CT, MRI, and EEG both post-trauma and after one year. This patient had a skull fracture and showed full recovery. Patient $\mathrm{C}$ had abnormal CoPET, CT, and MRI. The Co-PET showed cobalt accumulation in the left tempoal region that was 3.7 times the activity in the mirror region (figure). The EEG disturbances persisted throughout the follow up period of one year. This patient had several residual complaints after one year. Patient D showed structural abnormalities on CT, including a skull 
Clinical, CT, EEG, MRI, and Co-PET data on five patients (A-E) with closed traumatic brain injury

\begin{tabular}{|c|c|c|c|c|c|c|c|c|c|}
\hline Patient & GCS & $P T A$ & GOS & $\begin{array}{l}\text { CT } \\
\text { localisation }\end{array}$ & $\begin{array}{l}E E G \\
\text { localisation }\end{array}$ & $\begin{array}{l}\text { MRI } \\
\text { localisation }\end{array}$ & $\begin{array}{l}\text { Co-PET } \\
\text { localisation }\end{array}$ & $C E R$ & $C C L$ \\
\hline $\mathrm{A}$ & 10 & 5 & 7 & NA & $\begin{array}{l}\text { Right temporal- } \\
\text { occipital }\end{array}$ & NA & Right temporal & $2 \cdot 3$ & 354 \\
\hline \multirow[t]{2}{*}{ B } & 14 & 8 & 8 & & Left temporal & Left frontal & Left temporal & 3.0 & 876 \\
\hline & & & & NA & $\begin{array}{l}\text { Right temporal- } \\
\text { occipital }\end{array}$ & Right frontal & Right frontal & $4 \cdot 0$ & 4208 \\
\hline C & 11 & $\begin{array}{l}9 \\
7\end{array}$ & $\begin{array}{l}6 \\
8\end{array}$ & Left temporal & Left temporal & Left temporal & Left temporal & $\begin{array}{l}3 \cdot 7 \\
4 \cdot 0\end{array}$ & $\begin{array}{r}1506 \\
828\end{array}$ \\
\hline$\stackrel{D}{E}$ & $\begin{array}{r}10 \\
9\end{array}$ & 13 & $\begin{array}{l}8 \\
7\end{array}$ & Right temporal & Right temporal & $\begin{array}{l}\text { Right frontal- } \\
\text { temporal }\end{array}$ & Right temporal & $2 \cdot 9$ & 450 \\
\hline
\end{tabular}

GCS = Glasgow coma scale for assessment of level of consciousness with a total severity score from 3-15 (day of admission); PTA = post traumatic amnesia (days); GOS = extended version of the Glasgow outcome scale on a score from 0 (poor outcome) PTA = post traumatic amnesia (days); GOS = extended version of the Glasgow outcome scale on a score from 0 (poor outcome) to 8 (excellent outcome) (one year after traumatic brain injury); CT was on day of admission; EEG was within one week of traumatic brain injury; MRI and Co-PET were between three and four weeks after traumatic brain injury; CER = cobalt enhancement ratio (quotient of the mean average cobalt uptake in arbitrary units in the injured tissue $v$ the non-affected mirror brain); CCL $=$ cobalt contusion load: representing the total amount of radioactivity accumulated in an affected volume of the brain as the ratio of radioactivity in the same volume in the contralateral part of the brain; NA = no abnormalities

fracture. Both Co-PET and EEG showed abnormalities that were not in accordance with CT findings. The follow up EEG was normal and the patient recovered well. Patient $\mathrm{E}$ had abnormalities on Co-PET, CT, and MRI. The EEG findings were abnormal both post-trauma and on follow up. This patient was admitted to a psychiatric hospital.

\section{Discussion}

All patients made a fairly good recovery in terms of both GOS and neuropsychological testing. In all patients, neuropsychological testing (baseline), Co-PET, and EEG (after trauma) showed focal abnormalities in the affected frontal and temporal brain regions. By contrast, CT and MRI did not detect lesions in every case. Thus the lack of (structural) abnormalities on CT or MRI does not rule out the presence of (transient functional) brain damage (EEG; Co-PET) and consequent inpairment in neuropsychological testing, as seen in patients $A$ and $D$. In these two patients the abnormalities on EEG disappeared after one year and the outcome of the patients was good. The other three patients $(\mathrm{B}, \mathrm{C}, \mathrm{E})$ had persistent EEG abnormalities and initial structural damage on CT and MRI. Patients $C$ and $\mathrm{E}$ had a suboptimal outcome in clinical terms. Patients B and D both had a skull fracture, which may relate to their good outcome despite evident abnormalities on several imaging modalities.

There was no relation between neuroimaging data and clinical data (table) or neuropsychological testing data, either quantitatively or on localisation. Although all patients had frontal and temporal brain lesions, these were not those expected from the neuropsychological testing profiles. Apart from the limited sample size of our pilot study, the lack of a relation could be because of the broadly distributed cerebral representation of many cognitive functions (except perhaps memory). ${ }^{3}$ The neuropsychological testing battery as applied here is in fact not meant to localise lesions, but instead is aimed at the most common cognitive impairments resulting from traumatic brain injury, functions that have a limited localisation value.

Secondary (ischaemic) injury after trau- matic brain injury is a delayed phenomenon triggered by the primary (mechanical) injury, that begins a few hours after trauma and progresses over several days. The tolerance of neurons for primary injury is variable and depends on site and size of the impact and the mechanics of trauma. Secondary injury may rather be determined by residual rCBF, duration of ischaemia, and selective ischaemic vulnerability, differing from neuron to neuron and mediated through calcium channels. The fate of contusional ischaemic tissue (postischaemic recovery $v$ progressive metabolic derangement) is thus determined by both rCBF and calcium influx. Functionally impaired but potentially viable injured tissue is characterised by increased calcium influx up to 48 hours after traumatic brain injury. Thereafter, the calcium mediated inflammatory process may become an important pathogenic factor. The infiltration of these processes may characterise the contusional tissue up to five weeks after onset of traumatic brain injury. Thus cobalt may both be a measure for calcium mediated (ir)reversible damage to neurons and a marker for inflammation. Cobalt in blood exists in a free unbound form, in a protein bound form (mostly), and in a cell bound form (leucocytes). The basic (hypothetical) mechanism of detection in Co-PET is extravasation of intravenous cobalt into the affected brain areas, in either free (intact blood-brain barrier) or protein-bound form (disrupted blood-brain barrier). In addition, cobalt accumulation can be due to leucocyte infiltration into the affected area and the process of new capillary formation, creating a newly formed "cobalt blood pool."

In conclusion, Co-PET is able to show the site and size of brain damage in patients with moderate traumatic brain injury. These abnormalities may be either functional or structural. It may confirm and especially localise EEG findings. Also, Co-PET may confirm findings from CT or MRI. To determine whether there is any additional diagnostic or prognostic value and to ascertain the most appropriate time window after injury appropriate for monitoring ischaemic damage needs further study in a larger series of patients. It would be of interest to perform serial Co-PET at regular intervals, to add rCBF imaging modalities in 
combination with FDG-PET, and to use a neuropsychological testing battery suited for localisation.

We thank Cygne BV for providing ${ }^{55} \mathrm{CoCl}_{2}$. Our special thanks to Mrs J Venema for secretarial assistance. This work was supported by a grant (GGN 22.2741) from the Dutch Technology Foundation (STW)

1 Miller JD. Head injury. $\mathcal{F}$ Neurol Neurosurg Psychiatry 1993; 56:440-7.

2 Teasdale G, Jennett B. Assessment of coma and impaired consciousness. A practical scale. Lancet 1974;ii:81-3.

3 Van Zomeren AH, Van den Burg W. Residual complaints of patients two years after severe head injury. $\mathcal{f}$ Neurol of patients two years after severe
Neurosurg Psychiatry 1985;48:21-8.

4 Newton MR, Greenwood RJ, Britton KE, Charlesworth M, Nimmon CC, Carroll MJ, Dolke G. A study compar$M$, Nimmon CC, Carroll M, Dolke G. A study compar-
ing SPECT with CT and MRI after closed head injury. $f$ ing SPECT with CT and MRI after closed

5 Kao CH, Wang PY, Wang YL, Chang L, Wang SJ, Yeh $\mathrm{SH}$. A new prognostic index human cerebral infarcts by 99Tcm-HMPAO-labelled white blood cell brain SPECT. Nucl Med Comm 1991;12 1007-12.
6 Roper SN, Mena I, King WA, Schweitzer J, Garrett K, Mehringer CM, McBride D. An analysis of cerebral Mehringer CM, McBride D. An analysis of cerebral $99 \mathrm{~m}-\mathrm{HMPAO}$ SPECT and computed tomography. 7 Nucl Med 1991;32:1684-91.

7 Jacobs A, Put E, Ingels M, Bossuyt A. Prospective evaluation of technetium-99m-HMPAO SPECT in mild and moderate traumatic brain injury. $\mathcal{F} \mathrm{Nucl}$ Med $1994 ; 35$ :942-7.

8 Graham DI, Ford I, Hume Adams J, Doyle D, Teasdale GM, Lawrence AE, McLellan DR. Ischaemic brain damage is still common in fatal nonmissile head injury. $f$ Neurol Neurosurg Psychiatry 1989;52:346-50.

9 Ikeda Y, Long DM. The molecular basis of brain injury and brain edema: the role of oxygen free radicals. Neurosurgery 1990;27:1-11.

10 Apasov S, Redegeld F, Sitkovsky M. Cell-mediated cytotoxicity: contact and secreted factors. Curr Opin Immunol 1993;5:404-10.

11 Jansen HML, Pruim J, Vandevliet AM, Paans AMJ, Hew JM, Franssen EJF, et al. Visualization of damaged brain tissue after ischemic stroke with cobalt- 55 positron emistissue after ischemic stroke with cobalt-55 positror

12 Jansen HML, Willemsen ATM, Sinnige LGF, Paans AMJ, Hew JM, Franssen EJF, et al. Cobalt- 55 positron emission tomography in relapsing-progressive multiple sclerosion tomography in relapsing-prog

13 Jennett B, Bond M. Assessment of outcome after severe brain damage. A practical scale. Lancet 1975;3:480-4.

\section{NEUROLOGICAL STAMP}

\section{Thymus serpyllum (wild thyme)}

Wild thyme - a creeping shrub of the mint family - was, in former times, known as a valuable medicinal plant and as a "certain remedy for that troublesome complaint, the nightmare". It was also used to treat "headache due to inebriation" and "nervous disorders". The plant contains thymol, which has been used as a disinfectant and active ingredient for antiseptics, mouthwashes, and gargles. It also produces a lemony scent - a discovery made by ancient Athenian men-who made themselves elegant by rubbing their chest with a thyme scented lotion after bathing. In medieval times thyme mixed with honey was considered a potent remedy for pulmonary diseases. Inhalation of the herb was regarded as a remedy for melancholy and epilepsy.

It is shown in a series of stamps depicting medicinal plants issued by Bulgaria in 1966 (Stanley Gibbons 1858, Scott 1735).

L F HAAS

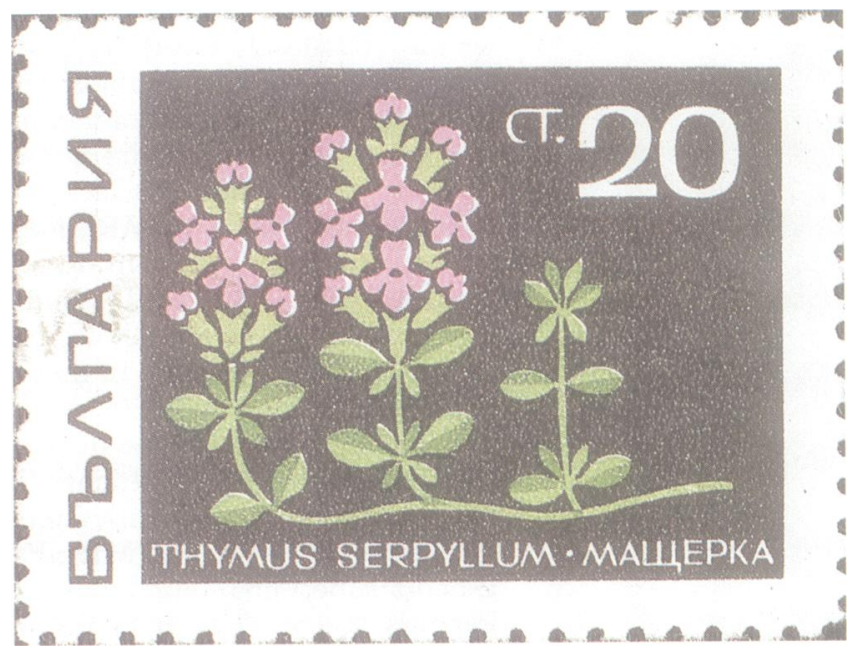

